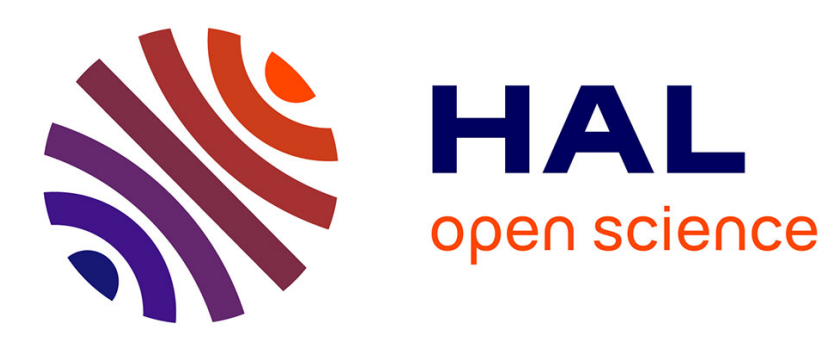

\title{
L'action des magistrats dans la régulation des risques collectifs : l'exemple des sports de montagne
}

\author{
Frédéric Caille
}

\section{To cite this version:}

Frédéric Caille. L'action des magistrats dans la régulation des risques collectifs : l'exemple des sports de montagne. Droit et Société, 2000, Justice et Politique (III). Les magistratures sociales, 44-45, pp.179-197. halshs-01358340

\section{HAL Id: halshs-01358340 \\ https://shs.hal.science/halshs-01358340}

Submitted on 31 Aug 2016

HAL is a multi-disciplinary open access archive for the deposit and dissemination of scientific research documents, whether they are published or not. The documents may come from teaching and research institutions in France or abroad, or from public or private research centers.
L'archive ouverte pluridisciplinaire HAL, est destinée au dépôt et à la diffusion de documents scientifiques de niveau recherche, publiés ou non, émanant des établissements d'enseignement et de recherche français ou étrangers, des laboratoires publics ou privés. 


\title{
L'action des magistrats dans la régulation des risques collectifs : l'exemple des sports de montagne
}

\section{Frédéric Caille}

\begin{abstract}
Magistrates' Actions in the Regulation of Risks : Mountain Sports as an Example.

This article, based on a large set of interviews, describes recent French legal developments and controversies in risks, related to the practice of mountain sports. These controversies concern both the liabilities of professionals (mountain and ski guides) and councils with winter resorts. The article investigates the social role and the autonomy of the professionals of justice in these matters, including their relationships with administrative authorities.
\end{abstract}

\section{Résumé}

Ce texte présente les controverses et transformations juridiques récentes concernant les risques collectifs liés à la pratique des sports de montagne. Bien que relevant de sphères juridiques distinctes (responsabilité des professionnels, responsabilité des stations), ces controverses sont perçues par les acteurs de terrain comme une totalité cohérente. Le texte présente les raisons objectives de cette convergence, avant d'interroger le rôle et l'autonomie d'action des professionnels de la justice en la matière. II s'arrête sur les logiques sociales, mais également judiciaires et administratives, qui concourent à la pénalisation du traitement collectif de ces risques particuliers, avant de revenir sur les formes de la catégorisation juridique et délictuelle elle-même.

\section{Citer ce document / Cite this document :}

Caille Frédéric. L'action des magistrats dans la régulation des risques collectifs : l'exemple des sports de montagne. In: Droit et société, n44-45, 2000. Justice et Politique (III). Les magistratures sociales. pp. 179-197;

doi : 10.3406/dreso.2000.1507

http://www.persee.fr/doc/dreso_0769-3362_2000_num_44_1_1507

Document généré le 06/06/2016 


\section{L'action des magistrats dans la régulation des risques collectifs : l'exemple des sports de montagne}

\section{Frédéric Caille *}

\section{Résumé}

Ce texte présente les controverses et transformations juridiques récentes concernant les risques collectifs liés à la pratique des sports de montagne. Bien que relevant de sphères juridiques distinctes (responsabilité des professionnels, responsabilité des stations), ces controverses sont perçues par les acteurs de terrain comme une totalité cohérente. Le texte présente les raisons objectives de cette convergence, avant d'interroger le rôle et l'autonomie d'action des professionnels de la justice en la matière. II s'arrête sur les logiques sociales, mais également judiciaires et administratives, qui concourent à la pénalisation du traitement collectif de ces risques particuliers, avant de revenir sur les formes de la catégorisation juridique et délictuelle elle-même.

Magistrature - Mise en danger d'autrui - Montagne - Politique pénale Responsabilité - Risques collectifs.

\section{Summary}

Magistrates' Actions in the Regulation of Risks: Mountain Sports as an Example

This article, based on a large set of interviews, describes recent French legal developments and controversies in risks, related to the practice of mountain sports. These controversies concern both the liabilities of professionals (mountain and ski guides) and councils with winter resorts. The article investigates the social role and the autonomy of the professionals of justice in these matters, including their relationships with administrative authorities.

Endangering other lives - Liability - Mountain - Penal policy - Public prosecutor - Risks.

\section{L'auteur}

Maître de conférences de science politique à l'Université de Haute-Alsace, membre du Centre européen de recherche sur le droit des catastrophes et accidents collectifs (CERDACC, Colmar) et chercheur associé au Centre de recherche sur le politique, l'administration, la ville et le territoire (CERAT, Institut d'études polítiques de Grenoble). Il poursuit des recherches sur les relations entre civisme et citoyenneté dans l'Europe des XIX et XX' siècles, ainsi que sur les nouveaux usages sociaux du droit en matière de gestion des risques collectifs.

Parmi ses récentes publications, on citera :

- " Le citoyen secoureur. Secours publics, sauveteurs et secouristes en France au XIX siècle ", Politix. Revue des sciences sociales du politique, 44, 1998.

* Université de Haute-Alsace, Faculté des Sciences Économiques et Sociales, 15 rue des Frères Lumière, F-68093 Mulhouse cedex. <fredcaille@hotmail.com> 
F. Caille

L'action des magistrats dans

la régulation des risques

collectifs : l'exemple des

sports de montagne
1. Louis ASSIER-ANDRIEU, Le droil dans les sociétés humaines, Paris, Nathan, 1996, p. 70.

2. Voir notamment Louis ASSIERANDRIEU, op. cit., p. 19 et suiv.

3. Jacques Commanle et JeanFrançois PERRIN, * Le modèle de Janus de la sociologie du droit ". Droit et Société, 1, 1985, p. 95-

112 ; Georges GURVTCH, L'expérience juridique et la philosophie pluraliste du droit, Paris, Pedone, 1935.

4. Paul AMSEnx, « Le droit, technique de direction publique des conduites humaines , Droits, 10 , 1989, p. 7-10.

5. La judiciarisation peut être envisagée comme l'aspect le plus saillant d'une juridicisation plus généralisée des modalités de régulation sociales et politiques, phénomène que, avec d'autres, le doyen Jean CARBONNIER identifie comme une véritable « passion du droit » désormais indissocia. ble sinon de notre conception, du moins de notre exercice de la République (Droit et passion du droit sous la V République, Paris, Flammarion, 1996).

6. Sur la redéfinition spécifique du rôle du droit dans la gestion sociale complexe des risques collectifs, voir notamment :

Marie-Angèle HERMITTE, Le sang et le droit. Essai sur la transfusion sanguine, Paris, Seuil, 1996. Plus largement : Jacques CAIL LOSSE, "Droit de crise, droit en crise ?", in Claude GIIBERT (sous la dir.), La catastrophe, l'élu et le préfet, Grenoble, PUG, 1990, p. 43-66; Claude LIENHARD, * Pour un droit des catastrophes $\#$, Recueil Dalloz, 1995, Chronique, p. 91.
* L'évolution juridique est avant tout le mouvement d'une idé ${ }^{1}$."

$\mathrm{Ni}$ totalement autonome, ni simplement assujetti aux fluctuations des rapports sociaux, le droit est par nature le lieu d'une incessante dialectique de la société sur elle-même. S'il prétend dire ce qui " doit être ", le droit reflète aussi ce qui est, cheminant entre l'expression d'une norme dont les critères prétendent à l'universalisme et l'application d'une règle de circonstance ${ }^{2}$. À ce titre, le droit et les usages sociaux dont il se trouve l'objet décrivent l'état d'une configuration socio-politique, en même temps qu'ils contribuent aux voies de sa transformation et de son devenir.

Ce statut ambivalent, ce "visage de Janus " de la juridicité ou de "l'expérience juridique " ${ }^{3}$, est sans doute le point de départ de toute réflexion sur la manière dont un espace social reçoit la formalisation juridique, y reconnait certaines de ses priorités ou de ses impératifs, et parfois s'y confronte. L'incursion du droit et de ses professionnels dans un tel espace social ne peut donc se comprendre dans le cadre strict de l'évolution propre à la raison juridique : elle témoigne aussi, et peut-être en premier lieu, du recours croissant à cet outil spécifique de direction publique des conduites humaines que sont les règles de droit ${ }^{4}$, et notamment de la judiciarisation comme modalité contemporaine de régulation des liens sociaux et politiques ${ }^{5}$.

On s'interrogera plus particulièrement ici sur le rôle du droit et des professionnels de la justice en matière de gestion sociale des risques collectifs liés à la pratique des activités sportives de pleine nature en montagne ${ }^{6}$. Fortement médiatisées, les mises en responsabilité juridiques de certains professionnels ou pratiquants de la montagne se présentent en effet depuis quelques années comme un débat central au sein de la configuration sociopolitique particulière au territoire alpin. De ce débat émerge notamment, et de manière récurrente chez quelques-uns des principaux acteurs concernés, la question de la place nouvelle que paraissent vouloir occuper les magistrats des juridictions de montagne. À l'image de "l'entrée dans le jeu " politique ou para-politique de certains professionnels de la justice, il semble que l'on ait affaire ici à la promotion de pratiques judiciaires novatrices, dont les visées propres rencontrent et favorisent les préoccupations d'autres acteurs sociaux ${ }^{7}$.

7. Cette perspective a notamment été développée par Violaine Roussel : « Les magistrats dans les scandales politiques ", Revue française de science politique, 48 (2), 1998, p. 245-273 ; et plus largement: Les magistrats dans les scandales politiques en France 1991-1997, Thèse de science politique, Université Paris X, janvier 1999. 
Au-delà de cette évolution des formes de régulation publique des activités de loisirs "à risques ", qui bouscule l'équilibre, le mode de "gestion paritaire " et les collusions d'intérêt traditionnelles caractérisant l'espace social des professionnels de la montagne $^{8}$, le caractère exemplaire du terrain d'investigation considéré tient à la manière dont y est reçue la norme juridique en tant que telle. La reconnaissance de sa légitimité s'affronte en effet aux fondements d'activités où la liberté de pratique et l'exposition au risque individuelles, ainsi que le rejet de toute forme de réglementation et de contrôle, participent de la définition de la pratique elle-même, et par voie de conséquence d'une forme d'auto-définition identitaire des pratiquants (alpinistes et guides de haute montagne notamment) ${ }^{9}$.

On choisira dans le présent texte de privilégier la perception de ces questions par les acteurs de terrain, à partir du matériel empirique recueilli 10 . On envisagera tout d'abord les controverses et transformations juridiques actuelles touchant à l'espace socioprofessionnel des activités sportives de pleine nature en montagne, controverses souvent mêlées, mais dont la dissociation préalable invite à mieux saisir les conditions de convergence. On reviendra ensuite sur les raisons de l'investissement nouveau des professionnels de la justice en la matière, par la présentation de certaines logiques sociales, mais également judiciaires et administratives, qui concourent désormais à la pénalisation du traitement collectif de ces risques collectifs particuliers. À ce titre, et in fine, les formes de la catégorisation juridique et délictuelle elle-même peuvent être interrogées, dès lors que c'est bien "la médiation opérée par la traduction en droit d'un événement (ou l'échec de cette tentative de qualification) qui fonde l'activité de justice, tant au plan symbolique que matériel ${ }^{11}$ ".

\section{Accidents de montagne et raison juridique}

La pratique des sports de montagne, pour l'amateur comme pour le professionnel, s'inscrit pour l'heure totalement dans le droit commun de la responsabilité civile, pénale ou administrative ${ }^{12}$. En dépit des espoirs et des argumentations des pratiquants, des organisations professionnelles ou des juristes spécialisés (lesquels, en dehors des magistrats, interviennent au titre de l'une ou l'autre des deux premières catégories), les activités de pleine nature en montagne ne bénéficient d'aucun traitement juridique spécifique.

Derrière son apparente banalité, cette inscription dans la généralité des règles de droit reflète immédiatement le défi structurel que rencontre cet espace social, dont les agents spécialisés revendiquent et objectivent le particularisme dans les termes d'un " territoire " (à la fois géographique et socio-politique) aux fortes
Droit et Société 44/45-2000

8. La « gestion paritaire » entre pouvoirs publics, professionnels et élus locaux, sur laquelle on ne peut s'arrêter id, est considérée, notamment par les acteurs institutionnels les mieux informés du milieu, comme l'une des caractéristiques structurelles des espaces de montagne, que ce soit au niveau communal ou dans le cadre de la régulation nationale des activités sportives de pleine nature. Voir, plus largement, Françoise GERBAUX, La montagne en politique, Paris, L'Harmattan, 1994.

9. Sur « la signification des activités engagées par les acteurs dans leur vie personnelle ou professionnelle, leurs loisirs, pour aller à la rencontre du risque ou s'en protéger $n$, que l'on ne peut développer ici, voir David LE BRETON, La sociologie du risque, Paris, PUF, 1995.

10. Ce matériel se compose d'une quarantaine d'entretiens menés par les trois membres de l'équipe avec les principaux acteurs institutionnels du domaine, entretiens non enregistrés mais intégralement retranscrits, et dont l'anonymat a été préservé. Ce matériel comprend également la participation à sept tables rondes ou journées de réflexion publiques sur cette question depuis avril 1998 (auxquelles il conviendraient d'ajouter de nombreux articles et débats de la presse spécialisée), qui témoignent de l'intensité de la préoccupation collective en la matière.

11. Pierre LASCOUMES et Anne DEPAIGNE, * Catégoriser l'ordre public : la réforme du Code pénal français de 1992 ", Genèses, 27, 1997, p. 25.

12. La seule exception, qui dépasse le cadre de la montagne, concerne le droit de l'encadrement des mineurs lors d'activités extra-scolaires rémunérées. Voir, par exemple, Arnaud PINGUET, " L'encadrement des mineurs en milieu extrascolaire ", in Montagne et responsabilité, Actes de la Journée d'étude Magistrats-Club alpin français, Chambéry, 30 mai 1997, p. 30-35. 


\section{F. Caille}

L'action des magistrats dans la régulation des risques collectifs : l'exemple des sports de montagne

13. Cette opposition structurelle est soulignée dans de nombreux entretiens, et notamment par tous les acteurs au fait des termes précis de la situation juridique (par exemple, entretien hautfonctionnaire ministère de la Jeunesse et des Sports en charge des questions de montagne, $28 / 09 / 98$ ). Le succès de la formule tient sans doute en partie à son application indistincte aux diverses sphères juridiques, et notamment à la délicate régulation en matière touristique ou économique des collectivités territoriales : cf. Jacques MANESSE, "La montagne saisie par le droit ? ", Pouvoirs locaux, 11, 1991, p. 70.76.

14. Vice-président de tribunal civil, ancien juge d'instruction d'une juridiction alpine et pratiquant de l'alpinisme (entretien 12/07/99), pour la première citation. Substitut du procureur de la République de Paris, membre du comité juridique et des instances nationales du Club alpin français (débat public du 05/12/98), pour la seconde.

15. Rapport sur la réglementation des nouvelles activités de montagne, 1991, p. 4 (réalisé à la demande du préfet de l'isère et consultable au Centre de documentation de l'ENSA à Chamonix).

16. Déclaration de solidarité des guides, alpinistes, skieurs, navigateurs et kayakistes, suite à l'incarcération de Daniel Forté après l'avalanche ayant frappé un groupe scolaire aux Crôts, près de la station des Orres (Hautes-Alpes) le 23 janvier 1998 (11 victimes). Déclaration faite lors du $5^{*}$ "Trophée Mer-Montagne " réuni aux Orres du 26 au 30 janvier 1998, reproduite dans Vertical, 116, 1998, l'une des principales revues spécialisées. spécificités. Les professionnels du droit, quelle que soit leur position à l'égard des spécificités du " territoire alpin " et de l'application circonstanciée des normes juridiques dont il doit être l'objet, soulignent ainsi volontiers que, pour être un " espace de liberté ", la montagne n'en est pas pour autant une "zone de nondroit " 13 . De la sorte, si certains n'hésitent pas à souligner que « le discours de victimes des guides est inadmissible", d'autres s'étonnent simplement des craintes des pratiquants amateurs ou professionnels qui dénoncent un encadrement normatif, qui ne déroge pourtant en rien de "la loi générale " 14 . De fait, cette position tend désormais à être largement partagée, à l'exception, on y reviendra, des défenseurs résolus d'une liberté de pratique qui se définirait par principe comme opposition aux « contraintes de la vie citadine et professionnelle 15 ", et pour lesquels il demeure " indispensable que la nature conserve ses prérogatives, et que sa découverte ne soit jamais soumise au seul droit des hommes 16 ".

En termes strictement juridiques, les deux aménagements principaux qui se trouvent aujourd'hui envisagés et placés au cœur du débat permettent immédiatement de percevoir l'étroite interdépendance des diverses sphères du droit de la responsabilité en la matière, ainsi que les deux grands domaines d'application d'une judiciarisation effective ou redoutée de la montagne.

\section{I.1. Cadre juridique et aménagements envisagés concernant la responsabilité des personnes}

Le premier aménagement relève du droit des personnes et des règles de jugement jurisprudentielles des accidents de montagne. Il concerne prioritairement la qualification juridique du cadre d'application de la responsabilité civile des professionnels des métiers sportifs de la montagne (guides de haute montagne, accompagnateurs de moyenne montagne, moniteurs de ski évoluant en domaine hors piste, plus rarement moniteurs d'escalade), bien qu'il s'applique également aux bénévoles des clubs alpins ou aux amateurs.

La mise en responsabilité des professionnels de la montagne peut se formaliser juridiquement de deux manières. La première relève de la responsabilité civile professionnelle de nature quasi délictuelle (faute de négligence ou d'imprudence), que la procédure s'inscrive dans le cadre civil ou pénal. La seconde source du droit commun de la responsabilité civile peut également être retenue, à savoir la voie de la responsabilité contractuelle, de préférence à l'encontre de l'employeur d'un professionnel, et dans le cadre strict d'une obligation de moyen et non de résultat. En d'autres termes, aucun " contrat de sécurité " ne lie un professionnel et son client dans ce milieu incertain et soumis par définition au rè- 
gne de l'aléa et de la force majeure. Dans le cas d'atteinte à l'intégrité physique des personnes, l'objectif de l'action juridique est donc clairement, quel que soit en définitive le cadre de mise en cause retenu, d'évaluer les éléments de la faute d'un professionnel (articles 1382 et 1383 du Code civil), faute éventuellement modulable par l'évaluation des aléas et de la force majeure (son principal critère d'évaluation étant en l'occurrence "l'imprévisibilité "), voire le comportement de la victime (très rarement retenu).

L'émoi des professionnels doit se comprendre au vu des limites étroites de ces éléments exonératoires, et sur la base objective de l'exposition au danger indissociable de la pratique des sports de montagne. L'aménagement juridique revendiqué par les acteurs du milieu concerne en effet la notion " d'acceptation des risques", notion dont l'acception courante exprime parfaitement le sentiment des pratiquants, mais dont la traduction juridique est fermement rejetée en la matière par une jurisprudence constante. Pour aller à l'essentiel, et pour l'heure en effet, le champ d'application de "l'acceptation des risques " en tant qu'élément exonératoire autonome ne concerne que la responsabilité sans faute " du fait des choses dont on a la garde ", ainsi par exemple des risques qualifiés de normaux dans la pratique de certains sports impliquant l'utilisation d'un objet (tennis, pelote basque), et notamment d'un " objet " de locomotion (sports aériens, équestres, nautiques). En matière de sports alpins, et en contradiction frontale avec le sens commun des acteurs, cette notion est donc totalement inopérante. Le client d'un professionnel ne peut se voir opposer une quelconque limitation a priori de la responsabilité de ce dernier du fait de sa propre connaissance des réalités aléatoires du milieu alpin, et c'est avec rigueur que les juridictions vérifieront « l'obligation de moyen " en matière de sécurité à laquelle est soumis le professionnel ${ }^{17}$. Tout au plus certains jugements récents ont-ils employé la notion de "connaissance du risque", notion plus " pédagogique ", semble-t-il, à l'égard des parties civiles que tout à fait orthodoxe quant à la technique du jugement pénal, lequel doit toujours se positionner par rapport à la responsabilité du prévenu, et n'est nullement soumis à l'analyse du comportement de la victime ${ }^{18}$.

Très concrètement, en définitive, un guide de haute montagne sera jugé in abstracto par comparaison avec "le modèle du bon professionnel de la spécialité ", c'est-à-dire du point de vue de son obligation contractuelle de moyen en matière d'information et d'interprétation des conditions météorologiques ou du milieu (neige, gel), de mise à niveau technique, d'équipement... Dans le cadre de la responsabilité délictuelle, le professionnel comme l'amateur verront estimer in concreto le lien pouvant exister entre la faute qui leur est reprochée et le dommage produit, le plus sou-
17. Sur tous ces points, voir notamment : Denis ASTRUC, "L'acceptation des risques en montagne : un argument de façade $n$, in Montagne et responsabilité, op. cit., p. 20-29.

18. Intervention d'un avocat spécialisé sur le thème de la responsabilité de la victime en matière d'avalanche, et débat dans le cadre d'un stage de formation continue des magistrats à l'École nationale de ski et d'alpinisme (ENSA), 03/03/99. 
F. Caille

L'action des magistrats dans la régulation des risques collectifs : l'exemple des sports de montagne

19. Les experts agréés par les juridictions alpines en matière d'alpinisme se comptent sur les doigts d'une main, et très peu d'experts privés généralistes interviennent sur la question du comportement en la mattère. Les experts requis par les magistrats sont presque tous des salariés du ministère de la Jeunesse et des Sports, et appartiennent au corps enseignant de l'ENSA.

\section{L'appréciation in concreto} participe au demeurant également d'une forme de normalisation renforcée des pratiques et des techniques pour l'ensemble du collectif, dès lors que dans ce domaine une expertise est souvent sollicitée. On peut supposer, « à la limite ", que la " faute " d'un alpiniste amateur emmenant un partenaire moins expérimenté serait juridiquement établie dès lors qu'un expert-professeur de l'ENSA qualifierait d'erreur technique l'emploi d'un matériel ou d'un mode de progression ancien et abandonné par les professionnels.

21. C'est notamment en ce sens, d'après les archives de l'ensemble des affaires judiciaires closes concernant des guides que l'équipe a dépouillées, que l'avocat du Syndicat national des guides de montagne rend compte du résultat des jugements à ses employeurs. De même, cf. entretien avec l'avocat du Syndicat des moniteurs de ski français, 27/05/99. vent, en cas de dommages importants, sur la base d'une expertise judiciaire ${ }^{19}$.

La principale conséquence de cette perspective juridique est ici, par définition et surtout dans le premier cas, d'associer de fait l'ensemble d'un milieu socio-professionnel au devenir judiciaire de l'action visant l'un de ses membres ${ }^{20}$. Ainsi, même salariés d'un organisme de tourisme ou de loisirs, les guides ou moniteurs de ski conservent le statut juridique de travailleurs indépendants, et dans la presque totalité des affaires leur défense se trouve assurée par l'avocat habituel de leur syndicat professionnel, dont la préoccupation première dépasse le cas du seul individu incriminé et concerne l'orientation de la jurisprudence sur la profession ${ }^{21}$.

En ce sens, il convient de le souligner immédiatement, la dimension collective et socio-politique des logiques d'action de la magistrature est ici incontournable et immédiate, alors même que l'on se trouve dans le cadre de la responsabilité civile et pénale individuelle. La nature des avis dont s'entourera le juge et les experts qu'il sollicitera sont en effet au cœur de l'acceptabilité sociale, pour les acteurs professionnels ou amateurs du milieu, de la mise en responsabilité judiciaire et de ses effets sécuritaires potentiels. Allons plus loin: c'est bien l'ensemble d'une configuration sociale spécifique qui se trouve directement concernée, associant aussi bien les responsables de l'enquête et la compétence qu'on leur prête (gendarmes de haute montagne, qui sont tous guides, procureurs "spécialisés »), le juge d'instruction (compétence territoriale de la juridiction où le fait dommageable a été commis), les experts divers (spécialistes nivologiques, guidesexperts), que les responsables des centres départementaux de Météo France, le Syndicat national des guides de montagne ou des moniteurs de ski, et plus généralement un grand nombre des acteurs participants de la gestion de l'espace alpin (assureurs, professionnels du tourisme, responsables des remontées mécaniques, maires de station, etc.). On mesure d'autant mieux cette interdépendance des différents acteurs face à l'action judiciaire, et par voie de conséquence des sphères juridiques dont ils relèvent, en observant le second aménagement juridique placé au centre du débat.

\subsection{Cadre juridique et aménagements envisagés concernant la responsabilité publique}

Cet aménagement concerne la responsabilité des collectivités, et plus encore la responsabilité personnelle des maires en charge des pouvoirs de police au titre de ces dernières. Bien qu'il concerne l'ensemble des activités sportives de pleine nature (escalade, canyoning, via ferrata, spéléologie, etc.), son domaine 
d'application principal concerne les pratiques hivernales des sports de neige (ski et randonnées en raquettes). Fer de lance du tourisme alpin et de son économie (la France est leader mondial de la fabrication des équipements et des matériels), ces activités, revivifiées par l'enneigement correct des dernières années, la diversification des pratiques (raquettes) et l'engouement d'un jeune public en faveur des nouvelles glisses (snowboard), sont en effet directement confrontées à la sévérité des dommages physiques aux personnes dont leur succès s'accompagne (un peu plus d'une dizaine de morts et autant de blessés par avalanche en France durant l'hiver 1998-1999 dans le cadre de ces pratiques ${ }^{22}$ ). La complexité du dossier sécuritaire, outre les risques inhérents au milieu alpin déjà mentionnés, tient en l'occurrence à l'imbrication étroite des comportements individuels et des politiques ou équipements de nature collective. C'est cette imbrication, on y reviendra, qui permet de comprendre les conditions objectives favorisant l'investissement des magistrats sur la question, et qui pour certains le justifie.

Du point de vue des responsabilités collectives, le maire est en effet chargé « sous le contrôle administratif du représentant de l'État ", est-il nécessaire de le rappeler, du * soin de prévenir, par des précautions convenables, [...] les accidents et les fléaux calamiteux, [...] les avalanches ", et cela sur l'ensemble du territoire de la commune (Code général des collectivités territoriales, art. L. 2212-1 et 2). En matière de pistes de ski, il lui revient notamment de veiller à l'élaboration et à l'application d'un Plan d'intervention et de déclenchement des avalanches (PDA), ainsi qu'au signalement explicite des limites des zones sécurisées et des obstacles naturels leur étant immédiatement contigus. La responsabilité pénale du maire ne fait aucun doute en cas de mise en œuvre défectueuse du PIDA, en particulier en cas d'avalanche sur une piste balisée et ouverte, bien qu'une jurisprudence naissante ne retienne parfois que celle du directeur de station ou du chef de service des pistes. Modéré dans ses principes, le " devoir d'information " du premier magistrat de la commune est pour sa part d'une application beaucoup plus délicate, alors même que les nouvelles pratiques de glisse invitent le pratiquant à la recherche d'une neige " vierge " (non damée, et donc non sécurisée sauf déclenchement naturel pouvant atteindre piste, route ou habitation) et que de nombreuses stations en soulignent la possibilité comme l'un des éléments principaux de leur communication publicitaire.

L'évolution de la portée judiciaire de la responsabilité du maire est indissociable de cette transformation des pratiques, que l'évolution technologique et la communication publicitaire proposent et rendent désormais pour une part accessibles bien au-delà du public marginal des skieurs expérimentés et pratiquants de la montagne qui s'y intéressaient jusqu'alors ${ }^{23}$. Rêve de " fun " et de
22. Non comptés les 12 morts de l'avalanche de Montroc ayant emporté des habitations dans la vallée de Chamonix le 9 février 1999.

23. Des remarques similaires pourraient faites sur la randonnée en raquettes, facilement accessible et très populaire désormais, mais qui ouvre l'espace de la montagne hivernale à un public qui ne l'aurait jamais envisagé dans le cadre de l'apprentissage technique plus exigeant du ski de randonnée. 
F. Caille

L'action des magistrats dans la régulation des risques collectifs : l'exemple des sports de montagne

24. On ne peut développer ici les termes juridiques de ce débat d'une grande importance. L'une des contributions les plus récentes est l'insertion par les sénateurs dans le projet de loi sur la présomption d'innocence de dispositions de nature à limiter les mises en responsabilité pénale des élus locaux pour fautes non intentionnelles. Le président du Sénat lui-même a pris ses distances avec les formes de cette initiative, fermement critiquée par le ministère de la Justice et les magistrats (Le Monde, 12-13 septembre 1999, p. 7). La doctrine, au nom du respect de l'égalité devant la loi pénale, semble s'orienter dans le même sens : voir, par exemple, Serge PEITT, "La responsabilité pénale des agents publics et des élus ", Gazette du Palais, 314, 1999, p. 3-10.

25. En ce sens, cf. l'intervention d'un représentant de l'Association internationale Moutain Wilderness, chercheur en informatique, guide et écrivain, au cours de la table ronde " Faut-il interdire la montagne? ", SIG, mars 1999. liberté, la glisse nouvelle est par définition " hors pistes ", propulsant du coup la définition des limites du "domaine skiable " d'une station dans une arène complexe, et pour l'heure principalement juridique, où s'arbitrent la préoccupation sécuritaire des autorités publiques (et de certaines associations de victimes) et le poids des intérêts économiques locaux.

Bien que reprenant et développant la notion depuis longtemps retenue par la jurisprudence de "piste de fait ", c'est-à-dire de " cheminement habituellement emprunté par les skieurs " même en dehors d'un espace balisé, phénomène dont on a dit qu'il demeurait jusqu'à récemment marginal, la définition juridique du "domaine skiable " est aujourd'hui perçue comme une menace majeure par l'ensemble des professionnels. Elle retient en effet dans les limites de ce dernier, de manière extensive, " tout ce qui est gravitairement accessible à partir des remontées mécaniques en retournant à la station ", engageant du même coup potentiellement la responsabilité pénale du maire pour les obligations précédemment citées (information et signalisation, sécurisation) sur un territoire parfois extrêmement vaste, mais qui n'en constitue pas moins désormais un atout touristique de premier plan. Trop, ou trop peu, les aménagements juridiques envisagés s'orientent en l'occurrence dans une double direction.

La première, qui est celle que défendent les professionnels $d u$ tourisme alpin, s'inscrit dans le prolongement de la prise en compte de la spécificité du territoire montagnard et de ses risques inhérents. Elle vise au premier chef à limiter la responsabilité pénale des élus pour des faits non intentionnels en matière d'action publique locale, débat dont on sait qu'il dépasse aujourd'hui de beaucoup l'espace de la montagne ${ }^{24}$. La puissante Association des maires de stations françaises de sports d'hiver et d'été (AMSFHE), mais également - depuis la condamnation par le tribunal administratif de Grenoble d'exploitants de remontées mécaniques - le non moins puissant Syndicat national des téléphériques de France (SNTF) plaident donc dans le sens d'une réglementation du domaine alpin décalquée de celle qui régit le domaine maritime. L'objectif est en l'occurrence de préciser des critères de démarcation entre espace "de la montagne " et " domaine skiable communal ", ou encore, pour parler comme les pratiquants, entre le " terrain d'aventure " et les domaines aménagés ${ }^{25}$. Il s'agit en somme de distinguer le domaine municipal du domaine public, lequel, relevant alors de la compétence exclusive de l'Etat en matière de police, limite strictement les prérogatives des maires et leur responsabilité (Code général des collectivités territoriales, art. L.2213-23). Si, en mer, les pouvoirs de police du maire s'exercent jusqu'à 300 mètres du littoral, tout en conservant au-delà l'obligation de secours et d'information, la difficulté reste de définir des critères simples, clairs, et qui soient applicables de ma- 
nière équivalente au milieu de la montagne. Le ministère de l'Intérieur, mais également la cellule juridique établie depuis deux ans auprès de la direction des Affaires criminelles et des grâces du ministère de la Justice pour les questions de sécurité en montagne ont accepté de travailler sur cette proposition ${ }^{26}$.

La seconde voie envisagée de réforme " de fait " de la responsabilité pénale pour risque des maires de station est indiscutablement portée par les autorités publiques, au premier rang desquels préfets et magistrats. On hésitera à la qualifier de véritable " aménagement normatif " dans la mesure où, faute de s'affronter au problème de délimitation de l'espace sur lequel s'exerce la police de la collectivité en matière de sécurité, elle se présente comme porteuse d'un véritable retournement réglementarojudiciaire, retournement dont les adversaires, pratiquants, professionnels et maires de station y compris soulignent la dimension liberticide potentielle. Transférant le regard des paradoxes de la politique et du discours des gestionnaires d'équipements et des professionnels du ski au comportement du pratiquant, ce retournement est probablement l'indice le plus sûr de l'autonomie affirmée des logiques d'action des magistrats en ce domaine.

\section{Des initiatives administratives et judiciaires novatrices, ou les mirages de la pénalisation}

Le retournement-aménagement judiciaire et réglementaire radical évoqué ci-dessus a sans nul doute concrétisé les craintes les plus importantes des acteurs de la configuration alpine depuis quelques années. Il révèle cependant en premier lieu le rôle central qu'occupent désormais les professionnels de la justice dans l'évolution, que certains jugent incontournable, d'un milieu social et professionnel jaloux de son indépendance, et qui avait pour l'essentiel réussi jusqu'alors à rester le partenaire obligé et parfois unique de toute élaboration normative le concernant ${ }^{27}$. À ce titre, on l'aura compris, la judiciarisation (notamment pénale) du traitement des accidents de montagne, qu'elle concerne la responsabilité des professionnels des métiers sportifs ou celle des élus locaux, est perçue comme une totalité cohérente par le plus grand nombre des acteurs. Bien qu'elle concerne des sphères juridiques assez distinctes, on peut s'accorder, semble-t-il, à reconnaitre le bien-fondé de cette assertion, ne serait-ce que du fait de l'homologie des défis qui s'imposent au droit, sommé pour la première fois en la matière de tracer les frontières de la liberté et de la responsabilité individuelles dans l'exposition ludique et volontaire au risque alpin.
26. Sur tous ces points, voir : entretien secrétaire général de l'AMSFHE, 23/03/99; entretien magistrat détaché au ministère de la Justice, direction des Affaires Criminelles et des Grâces, chargé du suivi de la sécurité en montagne, 05/02/99; une évo. cation de ce débat dans Les $\mathrm{Ca}$ hiers du CSSM, 12, 1999, p. 3435.

27. On a dit que l'on ne peut s'attarder dans cette présentation sur cet aspect déterminant de la configuration d'acteurs propre au milieu de la montagne. On rappellera cependant que les parlementaires de montagne disposent notamment, outre l'AMSFHE déjà évoquée, de l'Association nationale des élus de montagne (ANEM, 150 à 200 parlementaires), ainsi que de "groupes montagne "à l'Assemblée nationale et au Sénat. Dans le domaine sportif, le Conseil supérieur des sports de montagne (CSSM), créé en 1983, et ses commissions permanentes (dont une commission juridique en cours de création) élaborent la réglementation avec l'ensemble des partenaires concernés (une trentaine d'institutions diverses). Son secrétariat général est établi sous la tutelle du ministère de la Jeunesse et des Sports à l'École nationale de ski et d'alpinisme à Chamonix, institution sans équivalent créée en 1948, qui dispose d'un im. portant centre de documentation et délivre les formations aux métiers sportifs de la montagne. Ces sports sont les seuls à bénéficier d'un chargé de mission spécifique (avec les handicapés physiques) au ministère de la Jeunesse et des Sports, basé à Chamonix, auquel s'ajoutent près de 300 postes budgétaires du même ministère dans les écoles de ski et fédérations de la montagne. 
F. Caille

L'action des magistrats dans la régulation des risques collectifs : l'exemple des sports de montagne
28. Dominique GAURIER, * Responsable mais pas coupable. Esquisse d'anatomie d'une responsabilité sans pénitence obligée ", Actes de la $11^{\text {e }}$ séance du séminaire du programme Risques collectifs et situations de crise, 23 avril 1998, p. 11-26.

29. Olivier ABEL, « La responsabilité incertaine ", Esprit, 11, 1994 , p. 24 ; et plus largement l'ensemble du dossier spécial "Les équivoques de la respon. sabilité " de ce numéro.

30. Magistrat à la cour d'appel de Grenoble, entretien

26/05/99.
Le retournement juridique évoqué peut être résumé dans l'articulation de prescriptions réglementaires totalement inédites, pouvant aller jusqu'à l'interdiction totale de pratique, et d'une incrimination pénale nouvelle, "la mise en danger délibérée de la personne d'autrui » (article 121-3, al. 2 du Code pénal). L'interprétation de l'autonomie d'action des magistrats dans cette novation judiciaire nécessite au préalable d'être replacée dans l'évolution des usages sociaux du cadre juridique décrit précédemment.

\section{II.1. Logiques sociales de la pénalisation}

Les événements juridiques qui affectent aujourd'hui le domaine de la montagne participent d'une utilisation massive de la notion de "responsabilité ", dont le constat s'impose dans les espaces sociaux les plus variés. Ainsi que l'expliquent les historiens du droit, la véritable "explosion idéologique " du thème, à peu près totalement ignoré du Code civil, ne date guère que de la seconde moitié du XIX ${ }^{e}$ siècle, le droit public ayant le premier envisagé l'intervention d'une "responsabilité pour risque ", où la recherche de l'imputation de la faute cède le pas à l'objectif de réparation des dommages. À compter des années 1990 seulement, " la Cour de cassation commence elle-même à admettre la nécessité impérieuse du devoir de réparation dans le domaine de la responsabilité civile et donc à faire supporter une responsabilité pour risque " ${ }^{28}$. L'évolution de la raison juridique qui oriente la règle de jugement, sur laquelle on ne peut s'étendre, vaut en l'occurrence, on le sait, comme indice d'une transformation profonde de la conception dominante, et à proprement parler politique, des interdépendances sociales. Loin de suivre la lettre du droit et les fictions subtiles dont elle use, cette conception engage toujours résolument, semble-t-il, la responsabilité du côté d'une "vision pénale du monde ", selon laquelle la réparation et ses mécanismes juridiques demeurent imparfaitement dissociés de la recherche obstinée d'une culpabilité humaine ${ }^{29}$.

Le domaine des accidents de montagne, au dire des professionnels du droit interrogés, est l'exemple type de la généralisation de ce phénomène. La pénalisation, affirme ainsi un magistrat de cour d'appel, est " une tendance lourde qui remet en cause les acquis de la distinction entre infraction volontaire et infraction involontaire [...], l'expression d'un vieil archaïsme : il faut un responsable à sacrifier 30 ". Plus encore, s'appuyant sur la spécificité juridique française qui autorise la victime, de son propre chef, à engager des poursuites pénales, le recours au tribunal correctionnel semble faire partie " des moyens de défense des personnes face aux évolutions mal maîtrisées et mal comprises de leur environnement, une compensation au sentiment d'insécurité ou au be- 
soin de sécurité qu'elles éprouvent 31 ". Le constat est largement partagé par les magistrats ou avocats spécialisés.

Ainsi, pour l'avocat du Syndicat national des guides de montagne, " on a l'impression que les poursuites ne sont pas faites pour la société, mais par la société pour les victimes, le côté expiatoire de la justice revenant en avant, comme une sorte de vendetta juridicisée ". Si les recours judiciaires ne représentent en la matière qu'environ $4 \%$ des accidents déclarés, il n'est pas, dans les gros sinistres, de victime qui désormais ne porte plainte, au civil ou au pénal ${ }^{32}$. Selon un magistrat de juridiction alpine, la tendance est d'autant plus affirmée que, "dans ce contexte de loisirs, les gens acceptent mal que le loisir devienne dangereux : il s'agit d'un des domaines où les victimes sont souvent les plus vindicatives ". Parfois, ajoute-t-il, «il s'agit de se déresponsabiliser soi-même de la faute commise " 33 . Cette dimension "d'exorcisme " d'une prise de risque personnelle imparfaitement envisagée marque notamment le domaine des accidents de ski de montagne, où elle se place en regard de la pression exercée par les clients sur le guide ou moniteur de ski pour bénéficier des conditions de pratique les plus avantageuses, la "poudre ", indissociable des nouvelles glisses contemporaines ${ }^{34}$.

Quel que soit le jugement porté sur ces diagnostics sévères, on doit s'accorder à reconnaittre la place nouvelle de l'arène judiciaire comme lieu de confrontation d'univers sociaux distincts, et dont les perceptions du risque alpin demeurent pour l'heure très largement inconciliables. Au milieu de pratiquants informés et familiers de la montagne s'est en effet substitué un public d'usagers beaucoup plus indifférenciés, séduits par le rêve d'aventure sécurisée d'une offre touristique massive, dont les professionnels sont les premiers promoteurs. Si ces derniers sont peutêtre " victimes de ce qu'ils ont eux-mêmes généré 35 ", notamment par la communication en direction des jeunes, ils demeurent cependant fortement heurtés par le travail de deuil ou d'exorcisme que supporte parfois l'action pénale, pour des risques qui leur sont familiers et les affectent au premier chef.

Ce qui se présente comme fatalisme face au « danger objectif " pour des acteurs dont la mortalité professionnelle est l'une des plus élevées ( 61 guides, soit plus de $6 \%$ de l'effectif moyen du Syndicat national des guides de montagne de 1973 à 1986, en pratique d'amateur ou rémunérée ${ }^{36}$ ) est perçu par les parents de victimes comme un " véritable pacte morbide ". Pour ces derniers en effet, les jeunes usagers nouveaux ne sont pas des «inconscient(e)s ", mais " simplement pas conscient(e)s de la dangerosité " de pratiques dont on vend l'accessibilité et l'image magnifiée, tout en taisant l'apprentissage long et difficile de l'estimation circonstanciée des risques ${ }^{37}$. Ni expiation, ni déresponsabilisation à

\section{Droit et Société 44/45-2000}

31. Ibid.

32. Entretien 16/06/99.

33. Vice-président de tribunal civil, ancien juge d'instruction d'une juridiction alpine et prati. quant de l'alpinisme, précité.

34. Avocat du Syndicat national des moniteurs de ski français, précité. On rappellera que le ski en neige poudreuse, objectif premier désormais de nombreux pratiquants, s'oppose à la règle de sécurité largement admise il

$y$ a moins d'une quinzaine d'années, et qui voulait que le ski de montagne ne se pratique qu'à compter de la transformation et de la stabilisation du manteau neigeux, à savoir à compter de la mi-avril le plus souvent.

35. Vice-président de tribunal civil, ancien juge d'instruction..., précité.

36. Philippe BOURDEAU, " Guides de haute montagne. Territoire et identité ", Revue de géographie alpine, $\mathrm{n}^{\prime}$ hors série, 1991, $\mathrm{p} .55$. Les accidents de ski de montagne sont la cause de $48 \%$ des décès en activité professionnelle.

37. Entretien vice-président de Génération Glisse Snowboard Protection, association créée en janvier 1997 par des parents d'enfants décédés en domaine skiable (sur pistes balisées ou " hors piste ") et qui rassemble désormais 600 adhérents (22/05/99). 
F. Caille

L'action des magistrats dans

la régulation des risques

collectifs : l'exemple des

sports de montagne

38. L'Association a ainsi récemment obtenu de la cour d'appel de Grenoble une réouverture d'enquête et la constitution de partie civile pour l'accident mortel de deux jeunes surfeurs dans une zone en dehors des pistes balisées mais soumise à PIDA, pour laquelle le tribunal de Gap avait prononcé un non-lieu.

39. Ces divers éléments sont avancés par certains professionnels de la justice dans les entretiens effectués. Sur le rôle des médias on renverra le lecteur à ses souvenirs du traitement des avalanches de l'hiver 1999, ou de l'accident des Crôts. Sur la facilité de mise en ouvre et la rapidité de jugement de la juridiction pénale (qui se prononce aussi on le sait sur les réparations civiles dès lors qu'elle est sollicitée), on rappellera qu'en France la procédure civile est accusatoire, ce qui implique que la victime et son conseil doivent apporter la preuve de la faute, quand la procédure pénale est inquisitoriale, ce qui met l'enquête et ses frais (expertise notamment) à la charge de l'État. Outre qu'elle se présente, selon certains magistrats, comme plus rétributive * matériellement et symboliquement " pour les avocats, la voie pénale bénéficie également de la liberté d'appel en droit français, à la différence du monde anglo-saxon où les difficultés de sa mise en ouvre facilitent une culture de la transaction entre parties sur une base jurisprudentielle. tout prix, la judiciarisation n'est conçue par ces intervenants nouveaux de la configuration alpine que comme l'instrument obligé de réforme d'un milieu socio-professionnel fermé, où les intérêts économiques et la solidarité priment sur la prise en compte des dysfonctionnements. Le sentiment s'impose, dès lors, "d'avancer en parallèle " avec l'action des magistrats ${ }^{38}$ :

" Auparavant, le monde de la montagne s'autogérait. Maintenant, les magistrats sont les seuls qui font progresser les choses. Les gens de la montagne ont tout gâché. Ils ont trop visé le commercial. Ils ont trop faits l'amalgame entre la haute-montagne et le domaine skiable. "

Bien qu'elle ne puisse probablement expliquer à elle seule la pénalisation renforcée du traitement des accidents de montagne, où la médiatisation, le contentieux assurantiel, sinon même les dimensions rémunératrices de la procédure pénale peuvent jouer un rôle ${ }^{39}$, l'évolution de la mentalité ou de la psychologie des usagers de la montagne en est donc en définitive un facteur déterminant, et ce d'autant plus qu'elle se trouve relayée par les logiques d'action de certains magistrats des juridictions alpines.

\section{II.2. Logiques administratives et judiciaires de la pénalisation}

Comme évoqué précédemment, l'aménagement-retournement juridique récent qui révèle certaines des logiques d'action déjà affirmées des magistrats en matière de risque alpin se résume en effet dans l'articulation étroite de prescriptions réglementaires et d'une qualification pénale nouvelle. En d'autres termes, cette novation juridique affecte simultanément les domaines étroitement corrélés en la matière de la responsabilité civile ou pénale des pratiquants et de la "responsabilité publique " (que l'on peut qualifier de la sorte quand bien même, on l'a vu, elle se traduit judiciairement par une mise en responsabilité pénale personnelle) des élus locaux ou gestionnaires d'équipements.

C'est dans le domaine de la responsabilité publique que prend place l'innovation la plus révélatrice de ce retournement, sous la forme de l'arrêté de "réglementation de la pratique des activités sportives hors piste " du préfet de la Haute-Savoie, daté du 13 février 1999, arrêté prenant effet le jour même et s'appliquant jusqu'au mercredi 17 février. Justifié, selon les termes de la lettre explicative transmise aux maires des trois arrondissements concernés le 17 février, par les " circonstances météorologiques et nivologiques exceptionnelles " de la semaine précédente et par "les dangers d'avalanches qu'elles ont engendrés ", cet arrêté, pris en vertu du pouvoir de substitution de l'autorité préfectorale en matière de police municipale " compte tenu de l'étendue territoriale 

teurs enneigés d'altitude pour la pratique sportive à partir d'installations de remontées mécaniques", hors des domaines skiables aménagés. L'émoi considérable du milieu alpin, sur lequel on ne peut s'étendre ${ }^{40}$, s'est en l'occurrence pleinement inscrit dans la forme du débat éthique déjà récurrent, sinon du "droit au risque ", du moins de celui à sa gestion personnelle dans un milieu dont l'incertitude vaudrait comme gage d'un "besoin d'aventure [...] nécessaire, exutoire d'une énergie mal dépensée, évasion salutaire hors des contraintes sociales toujours plus restrictives et réductrices ${ }^{41}$ ". Extrêmement passionnelle, cette initiative est aujourd'hui présentée comme suit par le préfet de la Haute-Savoie :

" Il est toujours très difficile pour un maire de prendre un tel arrêté, du fait des impératifs économiques, renforcés dans une situation de ce genre qui concerne plusieurs massifs. Je n'ai pas voulu jouer au pionnier, ni innover. Je ne suis pas un idéologue. Je n'ai pas eu l'ambition de m'opposer au discours de liberté sur la montagne. J'ai seulement entendu répondre à une situation exceptionnelle par une mesure exceptionnelle et provisoire. Il ne s'agit pas d'un éclair dans un ciel serein mais, à nouveau, d'une mesure exceptionnelle et provisoire. Je ne prétends ni changer la société, ni faire école ${ }^{42}$. "

En dépit des dénégations affirmées de son auteur, cet arrêté, totalement inédit en matière de montagne ${ }^{43}$, doit, semble-t-il, être considéré comme l'indice le plus certain de l'investissement et du volontarisme renforcés des autorités publiques sur la question de la sécurité en montagne, bousculant d'un trait de plume la frontière symbolique de "l'espace de liberté ", associé par ses pratiquants aux risques et contraintes de la géographie alpine. Au-delà de facteurs circonstanciels exceptionnels - une cinquantaine de morts dans les pays de l'arc alpin limitrophes durant cette période, un contexte d'attention médiatique très importante (12 morts dans l'avalanche naturelle sur les habitations de Montroc le 9 février) -, l'usage de l'instrument réglementaire se présente en la matière comme un véritable pis-aller, qui tranche sans la résoudre la question des critères de délimitation des espaces aménagés et du domaine de la montagne. Deux observations plaident en ce sens.

La première concerne l'opportunité contestable de l'utilisation d'un instrument juridique peu réactif face à un milieu naturel très rapidement évolutif. En l'occurrence, le dimanche 14 février se présenta comme la première journée permettant la remise en route d'équipements d'altitude fermés depuis plus d'une semaine, accentuant l'incompréhension de professionnels dont la compé-

43 (suite) les zones accessibles gravitairement depuis les remontées mécaniques et situées à l'aplomb de pistes balisées dès que les bulletins affichés en bas des pistes indiquaient un risque d'avalanche supérieur à $3 / 5$ sur l'échelle européenne.
40. Voir les nombreux débats, articles, prises de position dans les différents titres de la presse spécialisée à partir de mars 1999. Un autocollant, portant en grosses lettres de couleur « NON Ȧ LA MONTAGNE INTERDITE ", et en SOUStitTe « TROP REGLEMENTER, C'EST DERESPONSABUISER ", est devenu le symbole de la protestation. Largement diffusé par les revues Montagnes Magazine et Skieur Magazine, par certaines organisations professionnelles, il est encore joint dans le catalogue hiver 2000 des principaux magasins de sport de la vallée de Chamonix.

41. Bernard AMY, Pierre CHAPOUTOT et Claude GARDIEN, Manifeste : pour que l'alpinisme reste l'art de gravir les montagnes. Ce manifeste, sollicitant l'adhésion des pratiquants et l'envoi de leur signature au Club alpin français, a été publié par la revue Vertical dans son numéro de mars 1998 et repris dans diverses publications locales.

42. Entretien 01/09/99.

43. On notera, en toute rigueur, que deux cas d'arrêtés municipaux similaires avaient devancé l'initiative préfectorale, arrêtés qui demeuraient perçus comme des originalités déplacées et marginales par l'ensemble des acteurs institutionnels concernés, exception faite du parquet d'Albertville en Savoie. Ce dernier (voir note infra) diffusa le 5 janvier 1999 à tous les maires de station de Savoie l'arrêté du 31 décembre 1998 du maire de Modane interdisant, « en raison du très faible enneigement ", le ski hors piste * sur tout le domaine skiable de Valfréjus ". Le second arrêté, en date du 3 février 1999 par le maire des ContaminesMontjoie (Haute-Savoie), et portant sur un ensemble de " prescriptions relatives à la sécurité des pistes de ski alpin ", interdisait le ski hors piste dans 
F. Caille

L'action des magistrats dans la régulation des risques collectifs : l'exemple des sports de montagne

44. Les interprétations de l'événement peuvent être assez divergentes. « Bronca » d'une communauté chamoniarde extrêmement soudée - ainsi que l'admet de manière implicite le préfet de la Haute-Savoie, qui reconnaît son * agacement "à l'égard de l'attitude des Chamoniards et des soutiens médiatiques dont ils bénéficient - ou réaction légitime des acteurs d'une commune comportant de nombreux professionnels privés d'activité depuis plusieurs jours en plein coeur de la saison hivernale, et sommés de rester dans la vallée au jour de l'amélioration des conditions? 45. Au total, plus d'une vingtaine d'arrêtés municipaux spécifiques auront été pris en ce sens à la date du 2 mars 1999 (dossier fourni par la préfecture de Haute-Savoie).

46. Par exemple, arrêté du maire de Mont-Saxonnex (arrondissement de Bonneville) en date du 20 février 1999, commune dont le domaine skiable est extrêmement limité mais qui offre de vastes espaces de ski de randonnée: "Article 1 : La pratique des activités sportives est interdite en dehors du domaine skiable aménagé et ouvert au public [...]. Article 3 : Ces dispositions entreront immédiatement en vigueur et seront applicables jusqu'au 30 avril 1999. " tence première, est-il nécessaire de le rappeler, réside justement dans la capacité d'évaluation des pratiques envisageables. Refusant d'obtempérer à l'injonction préfectorale, et soumise de fait à une fermeture presque totale de ses installations de par l'étendue d'un domaine skiable presque totalement extérieur aux pistes balisées, la commune de Chamonix obtint de la sorte en moins d'une heure un arrêté spécifique et limité à quelques couloirs d'avalanches bien référencés ${ }^{44}$.

La seconde observation concerne les suites de cet arrêté inaugural, dans des communes ne disposant ni des compétences professionnelles indiscutables de la vallée de Chamonix (École nationale de ski et d'alpinisme, compagnie de guides prestigieuse, pisteurs-artificiers très expérimentés, etc.), ni de ses ressources politiques spécifiques. Bien que l'on ne puisse ici en présenter le relevé précis, de nombreux maires relayèrent en effet dans les semaines suivantes l'initiative préfectorale, comme les y invitait d'ailleurs la lettre de confirmation de sa clôture du 17 février ${ }^{45}$. Si certains prolongèrent parfois de manière assez surprenante la mesure, d'autres l'étendirent au domaine communal bien au-delà des espaces accessibles à partir des remontées mécaniques, concrétisant ainsi les craintes des usagers d'une protection prioritaire de la responsabilité publique potentielle des maires, au détriment de la liberté d'usage de l'espace alpin 46 .

Ces remarques, qui pointent les limites en termes de " sécurité publique " ou de gestion des risques collectifs de l'outil réglementaire en matière d'activités sportives de montagne, laissent encore échapper la logique juridique effective dont il participe. On avancera en l'occurrence que cette dernière ne peut être appréhendée indépendamment de l'action de certains parquets des juridictions alpines, laquelle paraît associer des stratégies individuelles conquérantes à l'attrait de la qualification délictuelle récente de mise en danger de la personne d'autrui.

Plusieurs des acteurs rencontrés, au premier rang desquels les professionnels de la justice, ont en effet souligné l'investissement différentiel des magistrats en matière de sécurité alpine, notamment dans un cadre hivernal où l'attention médiatique vient renforcer une portée socio-économique considérable. Les avocats familiers de ces questions, bien que reflétant l'opposition structurelle qui les place face à la magistrature, se disent ainsi choqués et parfois « abasourdis " par les certitudes en matière de prévisibilité accidentelle de certains procureurs ou présidents de cour, « jeunes magistrats qui jouent les sages alors qu'ils ne comptent pas dix ans d'expérience ". On retrouve alors la dénonciation commune de ces magistrats " qui s'érigent à tort en justiciers " et font le lit de la pénalisation, et dont seules la modération et la compétence reconnue des cours d'appel viennent limiter les dérives. De manière plus mesurée, mais récurrente dans divers entretiens, l'utilisation 
affirmée de la voie pénale par le parquet d'Albertville en Savoie est ainsi relevée, marquant indiscutablement la volonté d'un procureur et, surtout, de son substitut, à la fois médiatique et très investi sur la question, de participer directement de la configuration alpine.

Accumulant les coups d'éclat, ce dernier symbolise l'espace d'autonomie qu'ouvrent aux magistrats les débats et incertitudes juridico-politiques précédemment évoqués, participant régulièrement aux tables rondes et débats en la matière, favorisant également la venue en jugement dans les semaines précédant l'ouverture de la saison de ski d'affaires "emblématiques" où se trouvent impliqués guides, moniteurs de ski et autres pratiquants amateurs ou professionnels de la montagne, "des audiences à l'épate ", disent certains. La manière dont il définit publiquement les missions de la justice dans le domaine des accidents de montagne plaide d'ailleurs en ce sens, puisqu'aux objectifs de répression des imprudences et de contribution à la réparation des préjudices subis par les victimes, il ajoute explicitement celui de "l'œuvre de prévention " au travers de la valeur exemplaire d'une condamnation 47.

Prenant acte que ce magistrat a gagné " une réputation de pourfendeur des imprudents " et accordant parfois une "valeur pédagogique en direction du grand public » à de tels agissements, les acteurs du milieu alpin se montrent néanmoins unanimes à dénoncer des initiatives plus audacieuses. Ainsi en est-il de la diffusion le 14 décembre 1998 à l'ensemble des maires des stations de Savoie, "pour information et à toutes fins utiles", de la copie de l'arrêté de l'un d'entre eux, " qui recueille toute [son] approbation ", pour les inviter à prendre des arrêtés réglementant la sécurité dans le cadre des domaines skiables, afin de faciliter les actions pénales au titre du délit de " mise en danger d'autrui " (art. 223-1 du Code pénal). L'initiative sera reproduite dans les mêmes termes le 5 janvier suivant, en l'occurrence pour inviter à des arrêtés d'interdiction du ski hors piste " en cas de conditions météo ou de neige dangereuses " et pour permettre " la poursuite préventive " au titre de la même incrimination ${ }^{48}$. Réagissant directement auprès de la Chancellerie, l'Association des maires de stations françaises de sports d'hiver et d'été a dénoncé une remise en cause du principe de séparation des pouvoirs, trouvant en l'occurrence l'oreille de la direction des Affaires criminelles et des grâces, " très réservée " sur une initiative qui relève de la responsabilité du préfet et non de l'administration judiciaire (dans la mesure où il s'agit d'inciter les maires à l'exercice de leur pouvoir de police administrative) ${ }^{49}$. Plus excessifs, certains professionnels de la justice n'hésitent pas à parler de "situation ambiguë", " nombre de maires considérant qu'en prenant de tels arrêtés ils rendaient un petit service au procureur, qui saurait bien leur ren-
Droit et Société 44/45-2000

47. Intervention au Salon de l'aménagement en montagne, 1998. De même, entretien procureur et substitut d'Albertville, $22 / 12 / 99$.

48. Les deux lettres sont signées par le substitut. Lettre du parquet d'Albertville en date du 14 décembre 1998, qui donne copie de l'arrêté du 30 septembre du maire de St Martin de Belleville, lequel reprend les 10 * règles de bonne conduite " énoncées par la Fédération internationale de ski : « On ne peut dès lors qu'approuver l'initiative de ce jeune maire de 77 ans [...] et inviter (en toute indépendance) les responsables de sécurité que vous êtes, en vertu de votre pouvoir de police, à envisager de prendre des mesures similaires. » Lettre du 5 janvier 1999 (cf. note supra) : * Un bravo en conséquence pour la décision d'interdiction (non générale dans le temps et dans l'espace) de ce maire, le premier à ma connaissance en Savoie à avoir pris un tel arrêté, que je serais enclin à recommander (en toute indépendance) en cas de conditions météo ou de neige dangereuses, au moins dans des secteurs restreints particulièrement exposés à certains moments aux avalanches..."

49. Entretiens secrétaire général de l'AMSFSHE et magistrat chargé du suivi des accidents de montagne au ministère de la Justice, précités. 


\section{F. Caille}

L'action des magistrats dans la régulation des risques collectifs : l'exemple des sports de montagne
50. Entretien parquet

d'Albertville, précité. C'est ce même parquet qui a constitué le dossier des Journées d'études action publique et sports de montagne (Chambéry, 2-3 décembre 1999), lesquelles, dans le cadre de la formation permanente des magistrats, ont porté sur « La responsabilité des collectivités territoriales. Rapports des parquets avec les maires et préfets $\%$.

51. Vice-président de tribunal civil, ancien juge d'instruction..., précité.

52. Entretien magistrat chargé du suivi des accidents de montagne au ministère de la Justice, précité. Le risque s'étant, malheureusement, réalisé, Daniel Forté a été poursuivi pour les délits de blessures et homicide non volontaires, dont la mise en danger d'autrui constitue une circonstance aggravante. dre la pareille ". Nul besoin pourtant de supputer de telles collusions pour prendre acte de celle, très objective, qui associe de fait dans le cadre de la qualification délictuelle de mise en danger de la personne d'autrui les pouvoirs administratifs et judiciaires. Les initiatives du parquet d'Albertville, on l'aura compris, décrivent en effet en creux les logiques d'action d'une magistrature se positionnant en partenaire actif et déterminant de la gestion du risque alpin, et ayant réussi à trouver le soutien de l'administration préfectorale dans le tournant réglementaire précédemment évoqué. Bien que la Chancellerie souhaite que le parquet d'Albertville s'abstienne pour l'heure de renouveler l'initiative des "portés à connaissance " aux maires évoqués, elle n'a pas désavoué la politique pénale engagée, et le ministère de la Justice est, depuis avril 1999, membre de droit de la commission de l'information et de la sécurité du Conseil supérieur des sports de montagne, lequel envisage de se doter d'une " commission juridique " spécifique ${ }^{50}$.

Élaboré principalement dans la perspective de fournir un outil juridique de poursuites préventives en matière de circulation routière ou d'accidents du travail, le délit de mise en danger de la personne d'autrui tend désormais, selon certains magistrats, à s'appliquer de manière croissante et de plus en plus large, comme dans le domaine des accidents de ski, où cette évolution leur semble " extrêmement dangereuse " 51 . Si, pour l'heure, les décisions rendues sur cette base demeurent peu nombreuses, cette évolution concerne cependant de manière prioritaire l'incrimination pénale permettant l'engagement de poursuites, rencontrant une tendance à la pénalisation déjà présente on l'a dit dans l'opinion, et qu'elle paraît favoriser par une locution très proche du sens commun. Suite à la catastrophe des Crôts, le guide Daniel Forté a ainsi été inculpé sous ce chef, en dépit d'un fondement juridique en l'occurrence très aléatoire, puisqu'il n'avait nullement transgressé délibérément un règlement imposant une attitude précise ${ }^{52}$. Il est dès lors important de bien dissocier cette double portée de cette qualification délictuelle.

En ce qui concerne la règle de jugement, le délit de mise en danger de la personne d'autrui permet avant tout la condamnation "préventive " d'un comportement estimé dangereux (le parquet d'Albertville le qualifie de " délit-obstacle " permettant d'éviter la réalisation d'un délit, en l'occurrence celui de blessures ou homicide involontaires). S'il autorise une sanction indépendamment d'un accident et de ses conséquences, ce délit s'apprécie néanmoins de manière restrictive d'après tous ses éléments constitutifs, à savoir " violation manifestement délibérée d'une obligation particulière de prudence ou de sécurité imposée par la loi ou le règlement " (article 223-1 du Code pénal), mais également mise en danger effective (ce qui demeure parfois difficile à établir en dehors de toute conséquence avérée). Si un débat doctrinal a été vi- 
goureusement engagé dès l'adoption de cet article, considéré par certains comme "la résultante d'un mélange savant et d'une jonglerie audacieuse entre des concepts classiques de droit pénal et des préceptes modemes de politique criminelle sécuritaire », et dont la rédaction est "bâclée, imprécise et superfétatoire ", il est particulièrement significatif pour le présent propos de relever qu'il se poursuit aujourd'hui autour de la poursuite pénale des skieurs hors piste ${ }^{53}$. C'est notamment sur la question de "l'élément intentionnel ", indispensable pour toute incrimination criminelle ou délictuelle depuis l'entrée en vigueur des nouvelles dispositions du Code pénal (article 121-3 al. 1), que l'interprétation juridique se divise ${ }^{54}$. D'un mot, face à la cour d'appel de Grenoble, critiquée pour la forme juridique de son arrêt par les commentateurs mais familière des questions de montagne, qui a relaxé le 19 février 1999 des skieurs ayant enfreint un arrêté municipal d'interdiction par risque 4 d'avalanche au motif que "la connaissance de la présence d'autrui " et la "conscience qu'il serait nécessairement mis en danger par son comportement " était nécessaire à la réussite de la poursuite, la Cour de cassation a considéré, dans une affaire similaire, le 9 mars suivant, que l'élément intentionnel du délit était constitué par la seule infraction à un règlement municipal d'interdiction ${ }^{55}$. À la vue de cette jurisprudence sévère de la plus haute juridiction, qui reste à confirmer, on relèvera que l'opposition de principe des pratiquants et professionnels de la montagne à toute forme de réglementation, bien que basée sur des fondements extérieurs à la stricte raison juridique, se trouve en définitive, et non sans paradoxe, totalement justifiée...

Néanmoins, c'est du point de vue de l'incrimination elle-même et de la possibilité d'ouverture de poursuites que cette décision de la Cour de cassation participe sans doute au premier chef de la portée véritable du délit de mise en danger d'autrui en matière de risque alpin. Bien que prise postérieurement (le 9 mars), elle donne en effet enfin la logique sous-jacente de l'aménagementretournement juridique récent dont participent conjointement l'action offensive des parquets alpins et celle de l'administration préfectorale haut-savoyarde. Comme l'avoue le préfet concerné, bien que "non-idéologue ", il a perçu au-delà des aspects sécuritaires un enjeu qui touche à "l'État de droit ", ce dont l'a convaincu la consultation préalable du parquet de Bonneville (HauteSavoie), et ce dont le félicitera par voie de presse dès le lendemain celui d'Albertville : en cas d'infraction à un règlement l'ouverture des poursuites par le ministère public peut être immédiate, alors qu'en l'absence de dispositions réglementaires (et il n'existe pas pour l'heure de "Code du ski ou de la montagne »), le parquet se doit d'établir par les faits la mise en danger de la personne
53. Les citations sont extraites de * La responsabilité pénale du skieur hors piste qui déclenche une avalanche ", La Semaine juridique, 6 octobre 1999, II, 10171, note Patrick LE BAS, auquel on renverra pour la bibliographie sur la mise en danger d'autrui.

54. On trouvera une bonne présentation dans Jacques-Henri ROBERT, Droit pénal général, $\mathrm{Pa}$ ris, PUF, 1999, p. 307-311 et 315-318.

55. Voir, pour le commentaire de la décision de la cour d'appel de Grenoble du 19 février 1999 : "La responsabilité pénale du skieur hors piste... " , art. cité ; sur la même décision : Recueil Dalloz, 1999, Jurisp., p. 480, note Michel REDON ; sur la décision de la Cour de cassation : "Le déclenchement d'une avalanche par des skieurs hors piste ", La Semaine juridique, 27 octobre 1999, II, 10188, note Jean-Michel Do CARMo SILVA. 
F. Caille

L'action des magistrats dans la régulation des risques collectifs : l'exemple des sports de montagne
56. Entretien préfet de la HauteSavoie, précité. En fait, on l'aura compris, cette interprétation est juridiquement inexacte et minimise l'importance de l'existence d'une obligation réglementaire de sécurité particulière, puisque ce n'est pas seulement pour l'ouverture des poursuites, mais pour la constitution du délit luimême, que la jurisprudence présente exige l'existence d'une telle réglementation en matière de risque alpin. d'autrui ${ }^{56}$. Cette reconnaissance explicite d'un objectif de " facilitation de l'action pénale " démontre, par-delà la collusion des objectifs des administrations judiciaires et préfectorales, la réussite des logiques d'action les plus offensives de la magistrature en matière de risque alpin.

\section{Conclusion}

On se limitera, en guise de conclusion provisoire, à deux brèves remarques sur la portée du droit comme mode de régulation des risques collectifs en montagne.

La première peut être portée au crédit de l'univers juridique et de ses professionnels. Bien que l'on puisse s'interroger sur l'opportunité du recours à la norme juridique pour réguler des tensions relevant très largement des mécanismes de concertation politico-administratifs les plus courants, et dont le milieu de la montagne est abondamment pourvu, force est de reconnaître, dans l'esprit des parents de victimes précédemment cités, que l'action volontariste de la magistrature accélère et favorise l'évolution d'une configuration d'acteurs dans laquelle les rapports de force sont longtemps demeurés univoques. Heurtant des acteurs de terrain qui n'en saisissent qu'imparfaitement la portée (notamment les professionnels des métiers sportifs de la montagne), et en subissent parfois les excès, la judiciarisation est pour une part ici le levier d'une mise en débat public sans doute inévitable. A ce titre, les stratégies les plus personnelles de certains magistrats ne font qu'actualiser des logiques sociales de confrontation, notamment entre " usagers-victimes " de l'espace commercial du ski alpin et gestionnaires d'équipements, qui s'inscrivent au cœur des rapports sociaux actuels et évolutifs du territoire alpin.

A contrario, et à la charge de l'univers juridique, la pénalisation excessive des questions de sécurité en montagne ne peut qu'interpeller sur ses dérives liberticides potentielles. II est à craindre en effet, et cela est peut-être l'aspect le plus menaçant de la juridicisation des controverses concernant les sports de montagne pour leurs pratiquants, que la dispute publique des finalités des activités commerciales ne s'étende à celle des pratiques sportives elles-mêmes, activités sportives dangereuses, socialement marginales, et parfois mal comprises. Masquant sans les résoudre les enjeux de délimitation des domaines aménagés et de l'espace de la montagne, qui ordonnent en priorité les mises en responsabilité publique des maires de station, le tournant réglementaire et juridique présenté révèle une pente obscure de la raison juridique : en l'absence d'une redéfinition des frontières des engagements et des responsabilités, c'est en premier lieu par la pénalisation des comportements individuels que se régulera le risque alpin. Dénoncée par nombre de magistrats familiers des questions 
de montagne, cette évolution, par-delà l'attraction d'une incrimination telle que celle de "mise en danger de la personne d'autrui ", dont l'écho médiatique est capable de balayer les alternatives les plus mesurées de la raison juridique, reste encore soumise à la pluralité des logiques d'action de la magistrature et des professionnels du droit eux-mêmes ${ }^{57}$. Ce n'est alors pas le moindre des paradoxes que de constater l'intérêt objectif qui unit, dans la maîtrise des destinations de la formalisation juridique, l'ensemble des pratiquants et des acteurs territoriaux de la montagne aux mobilisations associatives de victimes les plus récentes ${ }^{58}$.

57. Ainsi, le parquet de Grenoble n'a nullement participé aux Journées d'études des $2-3$ décembre 1999.

58. Une première version de ce texte a été présentée dans l'atelier du congrès de l'Association française de science poli. tique : * Les logiques d'action des magistrats, entre la norme et l'interprétation de la norme ", Rennes, 28 septembre-1" ${ }^{\text {a }}$ octobre 1999. Le propos s'appuie sur un projet de recherche réalisé en collaboration avec Damien Deschamps (maître de conférences de science politique, Université de la Réunion) et Jean-Paul Zuanon (chargé de recherche FNSP, CERAT-Grenoble), et intitulé : Attributions de responsabilités et juridicisation de la gestion sociale des risques collectifs: l'exemple des professionnels de la montagne. Ce projet, dont le résultat définitif est en court d'achèvement, a été retenu au titre des actions soutenues par le programme " Risques collectifs et situations de crise » du CNRS, en juin 1998. 